J. Clin. Chem. Clin. Biochem.

Vol. 19,1981, pp. $13-15$

\title{
Zur Standardisierung von Hepatitis B-Immunglobulinen
}

\author{
Von H. Uthemann \\ Biotest-Serum-Institut GmbH, Frankfurt am Main
}

(Eingegangen am 29. Februar 1980)

Zusammenfassung: Titerbestimmungen von Hepatitis B-Immunglobulinen, die z. B. mit Radioimmunoassays oder mit Hämagglutinationstests durchgeführt werden, zeigen stets große Streuungen und sind immer dann nicht vergleichbar, wenn versäumt worden ist, im gleichen Testansatz ein Referenzpräparat mitzuführen, auf das die Ergebnisse bezogen werden können. Es wird eine einfache und sehr gut reproduzierbare radioimmunologische Methode beschrieben, bei der über die Berechnung der Regressionsgeraden der Titerreihe eines Referenzpräparates dessen Endtiter ermittelt wird, mit dem der $\mathrm{Anti}_{\mathrm{H}} \mathrm{HB}_{\mathrm{s}}$-Gehalt zu prüfender Präparate verglichen werden kann.

\section{Standardization of hepatitis B immunoglobulins}

Summary: Titer determinations of hepatitis B immunoglobulins, as performed for example with radioimmunoassays or with haemagglutination tests, always show wide deviations; if a reference preparation, on which the test results can be based, is not run simultaneously with the test, the results are not comparable. A simple and easily reproducible radioimmunological method is described: the regression line is calculated for the titer series of a reference preparation, whose end titer is established; the anti- $\mathrm{HB}_{\mathrm{s}}$ content of the preparations under test can then be compared.

\section{Einführung}

Eine große Zahl von Veröffentlichungen befaßt sich mit der Prophylaxe der Hepatitis B mit normalen oder mit Hyperimmunglobulinen bei besonders gefährdeten Personengruppen und nach vermuteter oder nachweislicher Inokulation von infektiösem Material (1-20). Die auf ihre Schutzwirkung geprüften Präparate unterscheiden sich in ihren Protein- und Anti-HB ${ }_{s}$-Konzentrationen.

Die Überlegenheit der Hyperimmunglobuline gegenüber normalen Immunglobulinen ohne nachweisbaren oder geringen, dem Durchseuchungsgrad der Bevölkerung

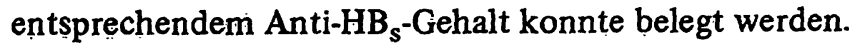

Zunächst beschränkte man sich darauf, die mit verschiedenen Meßmethoden ermittelten Konzentrationen des wirksamen Anti-HB als Endtiter anzugeben. Nun ist hinlänglich bekannt $(2,21)$ und durch umfangreiche eigene Untersuchungen bestätigt, daß Titerbestimmungen bei gleichen Präparaten, aber durchgeführt in verschiedenen Laboratorien und vielleicht sogar mit verschiedenen Methoden wie Radioimmuntest oder passive Hämagglutination, nicht vergleichbar sind, so daß die Forderung nach einem internationalen Standard für Hepatitis B-Immunglobulinpräparate berechtigt war und z. B. schon 1975 von Desmyter et al. erhoben worden ist (2). Diese Forderung ist erfullt, seitdem vom Bureau of Biologics (BOB) der Food and Drug Ad- ministration (FDA) (USA) ein Referenzpräparat, das auf einen im klinischen Versuch als wirksam erkannten Antikörpergehalt (100 IE/ml) eingestellt ist, zu exakten Vergleichsmessungen zur Verfügung gestellt werden kann. Nach Bonin (22) kann dieses Referenzpräparat so benutzt werden, daß Hepatitis B-Immunglobulinchargen mit gleichem oder höherem Antikörpergehalt freigegeben werden.

Voraussetzungen für vergleichende Untersuchungen auf der Basis des ,parallel line assay" sind neben sehr gut reproduzierbaren Meßverfahren genau einzuhaltende Versuchsbedingungen, besonders exakt anzusetzende Verdünnungsreihẹn, wofür klare Arbeitsanweisungen in den Prüflabors erarbeitet werden sollten (21). Wir setzen für Vergleichsmessungen von Gammaprotect ${ }^{\circledR}$ Hepatitis der Biotest-Serum-Institut GmbH Radioimmuntests ein und beziehen die Ergebnisse auf das Referenzpräparat Lot No. 2 des Bureau of Biologics der Food and Drug Administration.

\section{Material und Methoden}

\section{Radioimmuntests}

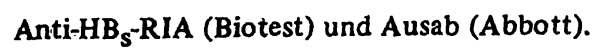

Verdünnungsmedium

Fötales Kälberserum (Seromed) 


\section{Referenzpröparat}

Hepatitis B-Immunglobulin des Bureau of Biologics (BOB), Lot No. 2, Food and Drug Administration (FDA) Bethesda, MD 20014, USA.

\section{Titration des Referenzpräparates}

Vom Referenzpräparat werden unabhängig voneinander zwei serologische Verdünnungsreihen in fötalem Kälberserum, das auch als Negativkontrolle verwendet wird, angesetzt, die ab den 1:1000-Verdünnungsstufen (also 1:1000, 1:2000, 1:4000 usw. bis 1:256000) getrennt getestet werden.

\section{Testbedingungen}

Die von den Herstellern der Radioimmuntests angegebenen Bedingungen und Auswertungshinweise werden exakt eingehalten.

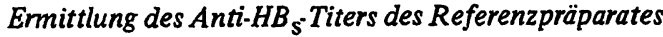

Jede Probe der zu prüfenden Verdünnungsstufen (je zwei) wird in einem geeigneten Gammacounter (23) dreimal gezählt, und aus den so erhaltenen sechs Meßwerten wird der Mittelwert gebildet, so daß sowohl Titrations- als auch Zählfehler weitgehend herausgemittelt werden. Die Logarithmen der so erhaltenen durchschnittlichen Zählraten ( $y$-Achse) und die entsprechenden Logarithmen der Verdünnungsstufen (ab 1:1000, $\mathrm{x}$-Achse) werden zur Ermittlung der Regressionsgeraden nach der Kleinstquadrate-Methode in einen technisch-wissenschaftlichen Rechner 2. B. vom Typ Texas Instruments TI-51-III eingegeben, wobei die Zählraten in Nähe oder unterhalb des Grenzwertes vernachlässigt werden, weil sich die Kurven dem Negativbereich asymptotisch nähern. Der Logarithmus des Endtiters des Referenzpräparates ergibt sich dann rechnerisch aus den Speicherwerten für die Regressionsgerade als Schnittpunkt zwischen dieser und der Grenzlinie. Aus dem gleichen Programm ist der Abschnitt auf der $\mathrm{y}$-Achse bei der 1:1000-Verdünnung als Logarithmus der Zählrate zu erhalten.

\section{Verdünnungen der Hyperimmunglobulin-Präparate}

Von dem zu vergleichenden Präparat werden unabhängig voneinander drei 1:1000-Verdünnungen in fötalem Kälberserum angesetzt, die getestet werden (24). Jede Probe wird dreimal gezählt, und aus den neun Meßwerten wird die durchschnittliche Zählrate des Präparates bei der Verdünnung 1:1000 ermittelt.

\section{Vergleich mit dem Referenzpräparat}

Die im Vergleich mit dem Referenzpräparat des Bureau of

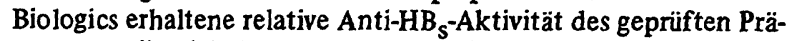
parates ergibt sich dann aus der Tatsache parallelen Kurvenverlaufs bei exakter Testdurchführung - Bonin spricht von einem echten Wertverhältnis zwischen zwei zu vergleichenden Präparaten durch Messung des Abstandes ihrer Dosis-CountsKurven im steilen Bereich (22) - wobei gilt:

$$
\begin{gathered}
\frac{\log \mathrm{cpm}_{\mathrm{BOB}}-\log \mathrm{cpm}_{\mathrm{Grenzwert}}}{\log \operatorname{Verdünnung}_{\mathrm{BOB}}-3,0}= \\
=\frac{\log \mathrm{cpm}_{(\mathrm{x})}-\log \mathrm{cpm}_{\mathrm{Grenzwert}}}{\log \text { Verdünnung }(\mathrm{x})-3,0} \\
\mathrm{cpm}=\text { counts } / \mathrm{min}=\mathrm{Imp} . / \mathrm{min}
\end{gathered}
$$

Nach Einsetzen der entsprechenden Werte und Umstellen nach (x) gibt der Antilogarithmus von $(x)+3,0$ den gesuchten und mit dem Referenzpräparat vergleichbaren Endtiter des geprüften Präparates wieder.

\section{Literatur}

1. Seeff, L. B., Wright, M. P. H., Zimmerman, H. J., Alter, J., Dietz, A. A., Felsher, B. F., Finkelstein, J. D., Garcia-Pont,

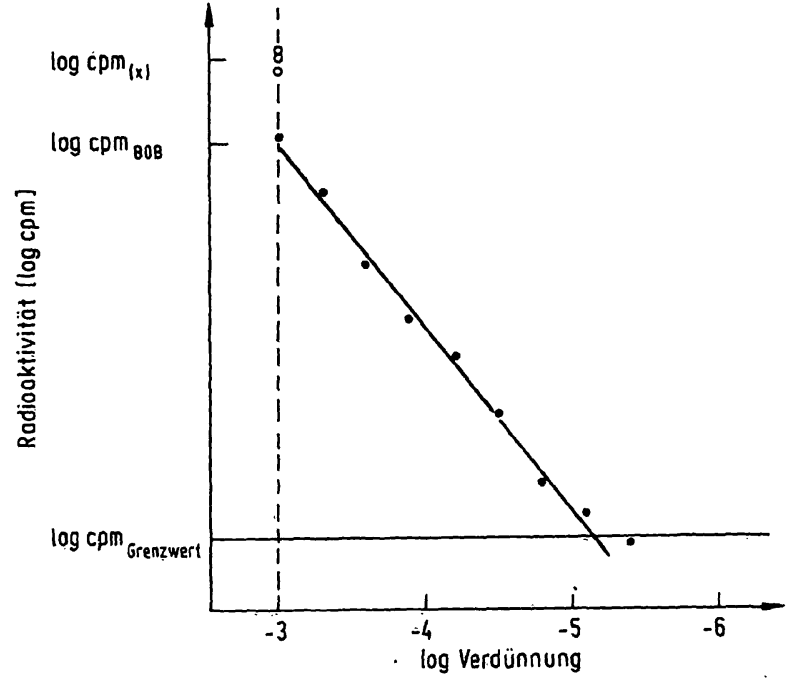

Abb. 1. Ermittlung des Anti-HB 5 -Titers. Vgl. auch Text.

\section{Ergebnisse}

Man muß sich dessen bewußt sein, daß keine curve fitting-Methode perfekt ist, und daß bei vergleichenden Untersuchungen Kompromisse gemacht werden müssen (25). Mit dem in der vorliegenden Arbeit beschriebenen Verfahren ist ein Vergleich von Hepatitis B-Immunglobulin-Präparaten mit einem Standard möglich, wobei die Unsicherheiten, die bei einfachen Endtiterbestimmungen auftreten, ausgeschlossen werden. Mehrfachtitrationen, die Ermittlung der durchschnittlichen Zählraten und das Zugrundelegen der Regressionsgeraden nach der Kleinstquadrate-Methode gestatten mit großer Näherung diesen Vergleich von Präparaten mit dem internationalen Standard des Bureau of Biologics oder anderen anerkannten Referenzpräparaten (Beispiele siehe Tab. 1).

Tab. 1. Vergleich von Hepatitis B-Immunglobulin-Präparaten mit dem Standard des Bureau of Biologies (BOB).

\begin{tabular}{llll}
\hline $\begin{array}{l}\text { Bestim- } \\
\text { mung }\end{array}$ & Präparat & Endtiter & Verhältnis \\
\hline 1. & BOB Lot No. 2 & $1: 78000$ & \\
& Gammaprotect 431119 & $1: 177600$ & $2,28 \times$ BOB \\
2. & BOB Lot No. 2 & $1: 38600$ & \\
& Gammaprotect 431 109 & $1: 169500$ & $4,39 \times$ BOB \\
3. & BOB Lot No. 2 & $1: 48000$ & \\
& Gammaprotect 431079 & $1: 153500$ & $3,20 \times$ BOB \\
\hline
\end{tabular}

P., Gerin, J. L., Greenlee, H. B., Hamilton, J., Holland, P. V., Kaplan, P. M., Kiernan, T., Koff, R. S., Leevy, C. M., 
McAuliffe, V. J., Nath, N., Purcell, R. H., Schiff, E. R., Schwartz, C. C., Tamburro, C. H., Vlahcevic, Z., Zemel, R. \& Zimmon, D. S. (1978), Ann. Int. Med. 88, 285-293.

2. Desmyter, J., Bradburne, A. F., Vermylen, C., Daneels, R. \& Boelaert, J. (1975), Lancet II, 377-379.

3. Prince, A. M., Szmuness, W., Mann, M. K., Vyas., G. N., Grady, G. F., Shapiro, F. L., Suki, W. N., Friedman, E. A., \& Stenzel, K. H. (1975), New Engl. J. Med. 293, 10631067.

4. Prince, A. M., Szmuness, W., Mann, M. K., Vyas, G. N., Grady, G. F., Shapiro, F. L., Suki, W. N., Friedman, E. A., Avram, M. M. \& Stenzel, K. H. (1978), J. Infect. Dis. 137, $131-144$.

5. Grady, G. F. \& Lee, V. A. (1975), New Engl. J. Med. 293, $1067-1070$.

6. Varma, R. R. (1976), J. Am. Med. Ass. 236, 2302-2304.

7. Bläker, F., Altrogge, H., Hellwege, H. H., Schliffke, C. \& Stephan, W. (1976), Dtsch. Med. Wochenschr. 101, 690693.

8. Deinhardt, F. (1978), Bundesgesundheitsbl. 21, 89-91.

9. Barandum, S., Skvaril, F. \& Morell, A. (1976), Schweiz. Med. Wochenschr. 106, 533-542, II. Teil 580-586.

10. Couroucé, A. M., Naret, C., Ciancionni, C. \& Delons, S. (1978), Lancet $I, 504$.

11. Seeff, L. B., Zimmerman, H. J., Wright, E. C., Felsher, B. F., Finkelstein, J. D., Garcia-Pont, P., Greenlee, H. B. Dietz, A. A., Hamilton, J., Koff, R. S., Leevy, C. M., Kiernan, T., Tamburro, C. H., Schiff, E. R., Vlahcevic, Z., Zemel, R., Zimmon, D. S. \& Nath, N. (1975), Lancet II, 939941.

12. Alter, H. J., Barker, L. F. \& Holland, P. V. (1975), New Engl. J. Med. 293, 1093-1094.

13. Szmuness, W., Prince, A. M., Goodman, M., Ehrich, C., Pick, R. \& Ansari, M. (1974), New Engl. J. Med. 290, 701706.
14. Couroucé-Pauty, A. M., Delons, S. \& Soulier, J. P. (1975), Amer. J. Med. Sci. 270, 375-383.

15. Delons, S., Kleinknecht, D. \& Couroucé, A. M. (1976), Lancet I, 204-205.

16. Ginsberg, A. L. (1976), Digest. Dis. 21, 404-408.

17. Hamilton, J. \& Iber, F. (1976), Digest. Dis. 21, 402-404.

18. Iwarson, S., Ahlman, J., Eriksson, E., Hermodsson, S. Kjellman, H., Ljunggren, C. \& Selander, D. (1977), J. Infect. Dis. 135, 473-477.

19. Seeff, L. B., Zimmerman, R. J., Wright, E. C., Finkelstein, J. D., Garcia-Pont, P., Greenlee, H. B., Dietz, A. A., Leevy, C. M., Tamburro, C. H., Schiff, E. R., Schimmel, E. M., Zemel, R., Zimmon, D. S. \& McCollum, R. W. (1977), Gastroenterology 72,111-121.

20. Prince, A. M., Szmuness, W., Woods, K. R. \& Grady, G. F. (1971), New Engl. J. Med. 285, 933.

21. Thomssen, R., Nationales Referenzzentrum für Virushepatitis am Hygiene-Institut der Universität Göttingen. Persönliche Mitteilung.

22. Bonin, O., Heymann, G., Schneider, W. \& Weeke-Lüttmann, M. (1978), Die gelben Hefte 18, 14-23.

23. Bales, Z. B., Patterson, J. F., Hoofnagle, J. H. \& Seeff, L. B. (1978), Transfusion 18, 91-93.

24. Bonomo, R. \& Stryker, M. (1978), Determination of potency of plasma samples containing antibody to hepatitis B surface antigen relative to a reference hepatitis B immune globulin. New York Blood Center, Quality Control Laboratory, Dec. 21.

25. Wilkins, T. A., Chadney, D. C., Bryant, J. \& Winder, R. L. (1977), Non-linear least sqares curve fitting of a simple theoretical model to radioimmunoassay dose-response data using a minicomputer. International Symposium on Radioimmunoassay and related Procedures in Medicine. Berlin, 31. 10. - 4. 11 .

Dr. H. Uthemann Biotest-Serum-Institut GmbH Postfach 730260 6000 Frankfurt 73 
. 